\title{
CT-like images based on T1 spoiled gradient-echo and ultra-short echo time MRI sequences for the assessment of vertebral fractures and degenerative bone changes of the spine
}

\author{
Benedikt J. Schwaiger ${ }^{1,2}$ (D) Charlotte Schneider ${ }^{1} \cdot$ Sophia Kronthaler ${ }^{1}$ • Florian T. Gassert ${ }^{1} \cdot$ Christof Böhm $^{1}$. \\ Daniela Pfeiffer ${ }^{1}$ - Thomas Baum ${ }^{2}$. Jan S. Kirschke ${ }^{2}$. Dimitrios C. Karampinos ${ }^{1}$ - Marcus R. Makowski ${ }^{1}$. \\ Klaus Woertler ${ }^{1} \cdot$ Markus Wurm $^{3} \cdot$ Alexandra S. Gersing $^{1}$
}

Received: 6 September 2020 / Revised: 28 October 2020 / Accepted: 3 December 2020 / Published online: 14 January 2021

(C) The Author(s) 2021

\begin{abstract}
Objectives To evaluate the performance of 3D T1w spoiled gradient-echo (T1SGRE) and ultra-short echo time (UTE) MRI sequences for the detection and assessment of vertebral fractures and degenerative bone changes compared with conventional CT. Methods Fractures $(n=44)$ and degenerative changes $(n=60$ spinal segments) were evaluated in 30 patients $(65 \pm 14$ years, 18 women) on CT and 3-T MRI, including CT-like images derived from T1SGRE and UTE. Two radiologists evaluated morphological features on both modalities: Genant and AO/Magerl classifications, anterior/posterior vertebral height, fracture age; disc height, neuroforaminal diameter, grades of spondylolisthesis, osteophytes, sclerosis, and facet joint degeneration. Diagnostic accuracy and agreement between MRI and CT and between radiologists were assessed using crosstabs, weighted $\kappa$, and intraclass correlation coefficients. Image quality was graded on a Likert scale.

Results For fracture detection, sensitivity, specificity, and accuracy were $0.95,0.98$, and 0.97 for T1SGRE and 0.91, 0.96, and 0.95 for UTE. Agreement between T1SGRE and CT was substantial to excellent (e.g., Genant: $\kappa, 0.92$ [95\% confidence interval, 0.83-1.00]; AO/Magerl: $\kappa, 0.90$ [0.76-1.00]; osteophytes: $\kappa, 0.91$ [0.82-1.00]; sclerosis: $\kappa, 0.68$ [0.48-0.88]; spondylolisthesis: ICCs, 0.99 [0.99-1.00]). Agreement between UTE and CT was lower, ranging from moderate (e.g., sclerosis: $\kappa, 0.43$ [0.26$0.60]$ ) to excellent (spondylolisthesis: ICC, 0.99 [0.99-1.00]). Inter-reader agreement was substantial to excellent (0.52-1.00), respectively, for all parameters. Median image quality of T1SGRE was rated significantly higher than that of UTE ( $p<0.001)$. Conclusions Morphologic assessment of bone pathologies of the spine using MRI was feasible and comparable to CT, with T1SGRE being more robust than UTE.

Key Points

- Vertebral fractures and degenerative bone changes can be assessed on CT-like MR images, with 3D T1w spoiled gradientecho-based images showing a high diagnostic accuracy and agreement with $C T$.

- This could enable MRI to precisely assess bone morphology, and 3D T1SGRE MRI sequences may substitute additional spinal CT examinations in the future.

- Image quality and robustness of T1SGRE sequences are higher than those of UTE MRI for the assessment of bone structures.
\end{abstract}

Keywords Magnetic resonance imaging $\cdot$ Spine $\cdot$ Degenerative intervertebral discs $\cdot$ Spinal fractures

Benedikt J. Schwaiger

benedikt.schwaiger@tum.de

1 Department of Diagnostic and Interventional Radiology, Klinikum rechts der Isar, School of Medicine, Technical University of Munich, Munich, Germany
2 Department of Diagnostic and Interventional Neuroradiology, Klinikum rechts der Isar, School of Medicine, Technical University of Munich, Munich, Germany

3 Department of Trauma Surgery, Klinikum rechts der Isar, School of Medicine, Technical University of Munich, Munich, Germany 


\section{Abbreviations}

T1SGRE T1-weighted spoiled gradient-echo

UTE Ultra-short echo time

ZTE Zero echo time

\section{Introduction}

Pathologies of the spine are among the greatest contributors to morbidity and mortality worldwide. In particular, degenerative changes such as intervertebral disc degeneration or degenerative spondylolisthesis have a lifetime prevalence of $60-90 \%$ [1-4]. Osteoporosis, either as a separate entity or in conjunction with degenerative changes, is another highly relevant pathology with a continuously increasing prevalence $[5,6]$. Ultimately, prevalent vertebral and hip fractures led to an increased risk of mortality 5 to 10 years after the fracture event, respectively $[7,8]$.

Both in patients with vertebral fractures and those with degenerative changes, CT and MR imaging are often performed [9]. In patients with fractures, this is done to differentiate acute from chronic fractures and to assess the surrounding soft tissues $[10,11]$. In patients with degenerative changes, this is done since pathologies of the spine - analogously to most other musculoskeletal entities - comprise both, a softtissue and an osseous component. CT examinations of the spine are associated with radiation exposure, additional examination time, and costs $[12,13]$.

Obtaining all relevant information on soft tissue as well as osseous structures within the same examination would therefore be highly desirable. While for the general detection of bone pathologies, e.g. cellular infiltration and sclerosis in bone metastasis, a fast and large field-of-view 3D T1w may be the best MRI sequence [14], high-resolution sequences with bone-specific signal characteristics may be the technique of choice for the precise assessment of bone morphology. For this, several approaches based on MRI have been previously suggested: "Simulated" CT images based on a 3D T1w fastfield echo MR sequence showed a high agreement with CT for the assessment of glenoid bone loss [15]. Similarly, it has been suggested that images resembling radiographs based on bone surface models derived from 3D MRI data allow for anatomic measurements in the ankle [16]. Recently, "simulated" radiographs and CT-like images were generated based on a 3D T1w spoiled gradient-echo MR sequence, in order to evaluate bone destruction patterns and periosteal reactions. The latter study showed that the accurate evaluation of bone tumors was possible using these images [17]. In other recent studies, the use of ultra-short echo time (UTE) and zero echo time (ZTE) sequences for the depiction of cortical and trabecular bone has been suggested [18-20]. UTE and ZTE sequences were successfully applied to other regions of the musculoskeletal system [21], but their application remains challenging in the spine due to folding and motion artifacts.
The purpose of this study therefore was to evaluate the diagnostic performance of MR-derived CT-like images based on high-resolution 3D T1w spoiled gradient-echo (T1SGRE) and ultra-short echo time (UTE) sequences for the identification and morphological assessment of vertebral fractures and degenerative bone changes in the thoracolumbar spine compared with conventional CT as a standard of reference.

\section{Methods}

\section{Patient selection}

An institutional review board (IRB) approval was obtained prior to this study (Ethics Commission, School of Medicine, Technical University of Munich, Germany). Patients gave their written informed consent.

Between December 2018 and October 2019, consecutive patients admitted to our emergency department were screened for study participation. Inclusion criteria were (i) admission for suspected acute thoracolumbar vertebral fracture, (ii) a CT acquired as part of the routine clinical diagnostic work-up, and (iii) feasibility of MR imaging within 3 days. Of 79 potentially eligible patients that were available for initial assessment regarding their participation in the study, 49 had to be excluded for the following reasons: 9 had contraindications for MR imaging (e.g., a pacemaker), 8 had undergone surgery immediately after the CT examination and therefore could not be examined with MR imaging, and 32 patients chose not to participate.

From our clinical information system, demographic data and clinical history including recent falls and symptoms were obtained.

\section{CT imaging}

CT was performed on one of two CT scanners (Somatom Definition AS+, Siemens Healthineers, and IQon Spectral CT, Philips) with the following parameters, according to routine clinical protocols: collimation, $0.6 \mathrm{~mm}$; pixel spacing, 0.4/0.3 mm; pitch factor, 0.8/0.9; tube voltage (peak), 120 $\mathrm{kV}$; modulated tube current, $102-132 \mathrm{~mA}$. Images were reformatted in 3-mm slice thickness using a bone-specific convolution kernel (I70H/YB).

\section{MR imaging and post-processing}

Within 3 days, MR imaging was performed on one 3-T scanner (Ingenia Elition, Philips) using 16-channel anterior and posterior coils. A 3D T1-weighted partial spoiled gradient echo (T1SGRE) and a 3D UTE sequence were added to the routine spine protocol (sequence parameters for T1SGRE and UTE, see Table 1). For this, a 3D UTE stack-of-stars sequence 
Table 1 Sequence parameters for 3D T1-weighted spoiled gradient echo (T1SGRE) and ultrashort echo (UTE) sequences

\begin{tabular}{|c|c|c|}
\hline & T1SGRE & UTE \\
\hline Echo time (ms) & 2.3 & 0.14 \\
\hline Repetition time (ms) & 7.8 & 6.3 \\
\hline Flip angle & $8^{\circ}$ & $5^{\circ}$ \\
\hline $\begin{array}{l}\text { Field of view (craniocaudal, } \\
\text { anterior-posterior, left-right; mm) }\end{array}$ & $250 \times 160 \times 70$ & $250 \times 259 \times 279$ \\
\hline Voxel size (acquisition; mm) & $0.45 \times .045 \times 1.5$ & $0.45 \times 0.45 \times 3$ \\
\hline Voxel size (reconstruction; mm) & $0.28 \times 0.28 \times 0.75$ & $0.28 \times 0.28 \times 0.75$ \\
\hline Parallel imaging & None & None \\
\hline Comment & $\begin{array}{l}\text { Partial Fourier imaging } \\
\text { in frequency encoding } \\
\text { direction }(60 \%)\end{array}$ & $\begin{array}{l}\text { 3D stack-of-stars sequence with } \\
\text { non-selective RF pulse and } \\
\text { Cartesian phase encoding in } \\
\text { the third dimension. }\end{array}$ \\
\hline $\begin{array}{l}\text { Acquisition duration } \\
\quad \text { (average } \pm \text { standard deviation; min) }\end{array}$ & $5.12 \pm 0.17$ & $6.3 \pm 0.23$ \\
\hline
\end{tabular}

was employed with a non-selective RF pulse and Cartesian phase encoding in the third dimension [22] in order to assess the signal of tissues with short $\mathrm{T} 2 *$. Of note, due to the hard RF pulse of the UTE sequence, a larger FOV in the slice direction had to be used to avoid folding artifacts. As soon as possible after the excitation, the FID was acquired in-plane along one center-out radial "spoke". The non-Cartesian trajectories were estimated using the gradient impulse response function of the system. For the reconstruction, an image reconstruction toolbox (ReconFrame, Gyrotools) was used to grid the data in two dimensions with the corresponding $\mathrm{k}$ space trajectories and to Fourier transform in 3D.

For the evaluation of osseous structures, both the 3D T1w GRE and UTE sequences were reformatted in sagittal, coronal, and axial orientation with a slice thickness of $3 \mathrm{~mm}$, grayscales were inverted, and windowing was set to resemble a CT bone window (Figs. 1 and 2).
According to our clinical standard for suspected vertebral fractures and degenerative changes, the protocol further comprised a sagittal short-tau inversion recovery (STIR) sequence, sagittal T1w and T2w spin-echo sequences, and a transversal T1w spin-echo sequence.

\section{Image analysis}

MR and CT images were individually and independently read by two radiologists (B.J.S., a board-certified radiologist with 10 years of experience in MSK imaging and F.T.G., a radiology resident), blinded to all other information including clinical and results from other modalities, including other MRI sequences when evaluation the bone-specific T1SGRE and UTE sequences. The images were read in a randomized order. Image evaluation and quantitative measurements were performed on a PACS cer-
Fig. 1 Comparison of T1SGREderived CT-like images (left), UTE images (middle), and conventional CT images (right) of the same patient. While the acute compression fracture of $\mathrm{L} 1$ (Genant ${ }^{\circ} \mathrm{I}, \mathrm{AO} /$ Magerl A1) can be identified and classified in all modalities; the T1SGRE sequence shows a more homogeneous signal and tissue contrast optimal for the assessment of osseous structures. Of note, due to the inverted-grayscale T1w contrast, ligaments are also shown as hyperintense which must not be misinterpreted as calcifications
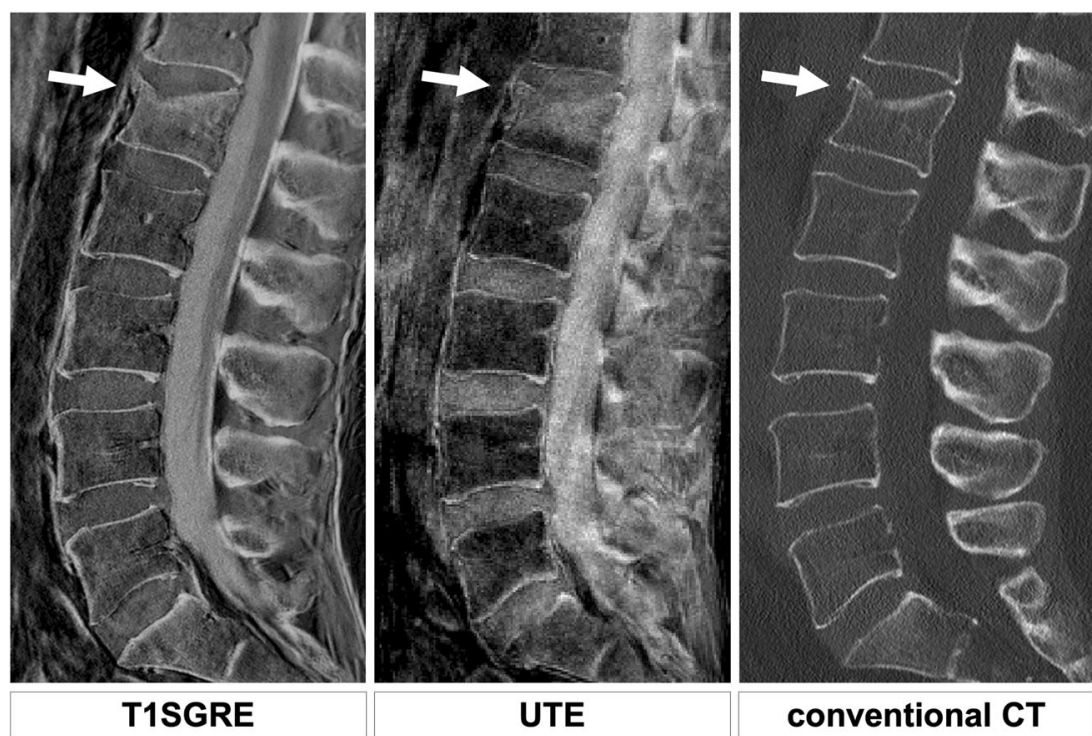


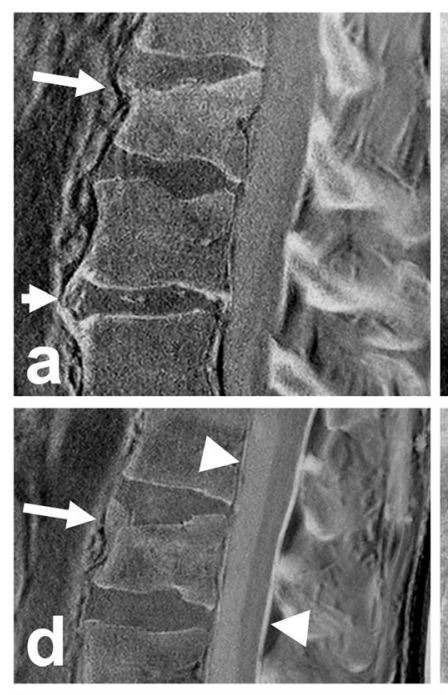

T1SGRE

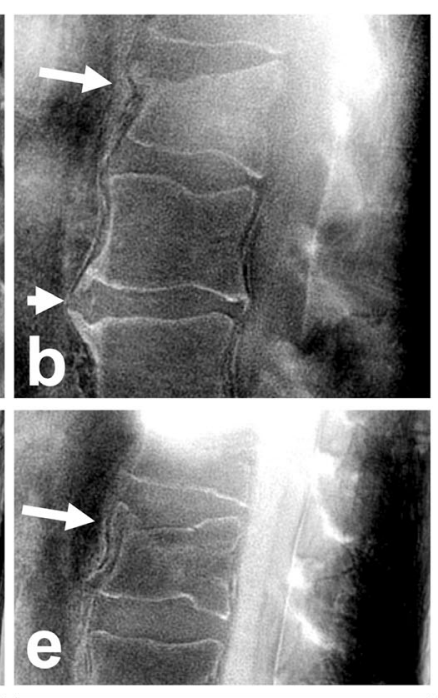

UTE

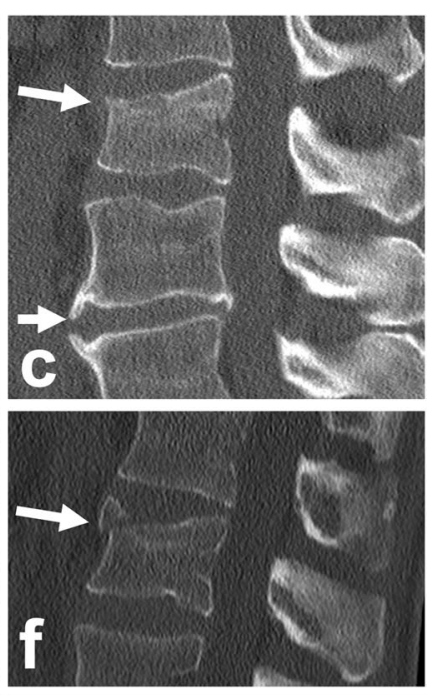

conventional CT
Fig. 2 Comparison of T1SGRE-derived CT-like images (a, d), UTE images (b, e), and conventional CT images $(\mathbf{c}, \mathbf{f})$. In one patient $(\mathbf{a}-\mathbf{c})$, a wedge-compression fracture of L1 with signs of an acute pathology such as a compaction zone can be depicted (upper arrows), as well as ventral and a small dorsal osteophytes on level L2/3 (lower arrows). In another patient $(\mathbf{d}-\mathbf{f})$, another wedge-compression fracture of $\mathrm{L} 2$ with a triangular teardrop-like fragment can be identified (arrows). Also note the thin hyperintense line running longitudinally along the posterior walls of vertebral bodies representing the posterior longitudinal ligament as well as the thicker hyperintense line posterior to the dural sac representing the ligamenta flava (arrowheads; d), which are not depicted on CT (f), and must not be misinterpreted as ligament calcifications tified for clinical use (IDS7 21.2, Sectra). Between T1SGRE, UTE, and CT imaging readings, there was an interval of at least 8 weeks, respectively.

First, the presence and location of vertebral fractures were assessed and noted. Then, the following morphological features were evaluated using a standard template (Table 2; Fig 3): height of the anterior and posterior vertebral edge in the mid-sagittal plane, height loss according to Genant et al [23], fracture classification according to $\mathrm{AO} /$ Magerl [24, 25], and differentiation of acute vs. chronic vertebral fractures according to Hedderich et al [26]. In patients with more than one fracture, each level was evaluated separately.

Furthermore, in every patient, in the two non-fractured segments with the most prominent degenerative changes (as determined by the more experienced radiologist, B.J.S.), the following imaging findings were evaluated (Table 2; Fig 3): anterior and posterior distance between bony endplates (i.e., intervertebral disc height) [27], anteroposterior (AP) diameter of intervertebral foramina [28], spondylolisthesis (distance between vertebral body rims, $\mathrm{mm}$ ), and extent of diffuse sclerosis adjacent to one or both vertebral endplates [29], of osteophyte formation [29], and of facet joint degeneration [30].

Images from all modalities were graded for overall diagnostic image quality on a five-point Likert scale (score of 1 , inadequate; 2 , poor; 3 , moderate; 4 , good; 5 , excellent).

\section{Statistical analysis}

In addition to descriptive statistics, the agreement of numerical, approximately normally distributed data was evaluated with intraclass correlation coefficients (ICC) and Bland-Altman plots were created for illustration purposes. The diagnostic performance of MRI for the detection of fractures was assessed using contingency tables. The agreement of ordinal scaled parameters was assessed using weighted Cohen's $\kappa$ [31]. To assess inter-reader reproducibility of the readings of MR-based CT-like images and CT images, the same tests were used. For all measures, 95\% confidence intervals (CI) were calculated. B.J.S. (11 years of experience in biostatistics) analyzed all data with SPSS, version 25 (IBM).

\section{Results \\ Patient characteristics, morphology, and image quality}

In total, 30 patients ( $65 \pm 14$ years; $60 \%$ female) with a total of 44 vertebral fractures (according to CT as the standard of reference) were included in this analysis, and on average, each patient had 1.5 vertebral fractures (range, 1-4). Of the fractures, 25 were considered acute according to the presence of 
Table 2 Imaging parameters for the assessment of vertebral fractures and degenerative changes

\begin{tabular}{ll} 
Parameter $\quad$ Description and references & $\begin{array}{l}\text { Grading and frequency distribution }(n, \%) \text { or mean } \pm \\
\text { standard deviation* }\end{array}$ \\
\hline
\end{tabular}

Fractures

Genant classification

Anterior height vertebral body

Posterior height vertebral body

$\mathrm{AO} /$ Magerl fracture classification

Fracture age

Degenerative changes

Anterior disc height

Posterior disc height

Neuroforaminal AP diameter

Spondylolisthesis

Osteophytes

Sclerosis

Facet joint degeneration
Semiquantitative visual grading of vertebral deformities according to Genant et al [23]

Measured in the median sagittal plane, from the anterosuperior to the anteroinferior corner of the vertebral body, excluding osteophytes, or dislocated fragments

Measured in the median sagittal plane, from the posterosuperior to the posteroinferior corner of the vertebral body, excluding osteophytes, or dislocated fragments

Classification of fractures in compression, distraction, and translation injuries according to Magerl et al [24] and Vaccaro et al [25]

Classification of fractures in acute and chronic fractures according to Hedderich et al [26]

Measured in the median sagittal plane, from the anteroinferior to the anterosuperior corner of the vertebral body, analogously to Frobin et al [27]

Measured in the median sagittal plane, from the posteroinferior to the posterosuperior corner of the vertebral body, analogously to Frobin et al [27]

Minimum AP diameter of the narrower side, measured in sagittal reformations according to Mamisch et al [28]

Maximum extent of listhesis, measured in the median sagittal plane

Anterior, lateral, and posterior osteophytes, assessed analogously to Wilke et al [29]

Extent of diffuse sclerosis in adjacent vertebral bodies, analogously to Wilke et al [29]

Extent of facet joint degeneration (if asymmetric, the more severe side was noted), according to Weishaupt et al [30]
Grade 1 (20-25\% reduction in height): $23(52 \%)$

Grade $2(25-40 \%): 12(27 \%)$

Grade 3 (> 40\%): 9 (21\%)

$19.4 \pm 5.5 \mathrm{~mm}$

$24.8 \pm 4.0 \mathrm{~mm}$

A1 (wedge compression): 30 (68\%)

A2 (split): 1 (2\%)

$\mathrm{A} 3+4$ (incomplete and complete burst): 13 (30\%)

$\mathrm{B}$ (distraction): 0

C (displacement or dislocation): 0

Definitely chronic: 14 (33\%)

Likely chronic: 1 (2\%)

Likely acute: $11(25 \%)$

Definitely acute: (40\%)

$10.7 \pm 5.6 \mathrm{~mm}$

$5.5 \pm 5.2 \mathrm{~mm}$

$9.2 \pm 2.0 \mathrm{~mm}$

$0.5 \pm 2.1 \mathrm{~mm}$

None: $24(41 \%)$

Mild: 25 (43\%)

Moderate: $8(14 \%)$

Severe: $1(2 \%)$

None: 38 (66\%)

Partially in one vertebra: 9 (16\%)

Partially in both vertebrae or encompassing the whole cross-sectional area in one vertebra: $9(16 \%)$

Encompassing the whole cross-sectional area in both vertebrae: $2(3 \%)$

Normal facets: 17 (29\%)

Joint space narrowing: 25 (43\%)

Plus sclerosis or hypertrophy: 15 (26\%)

Severe degeneration with narrowing, sclerosis and osteophytes: $1(2 \%)$

Plus synostosis: 0

\footnotetext{
*As shown by conventional CT as a standard of reference
} 


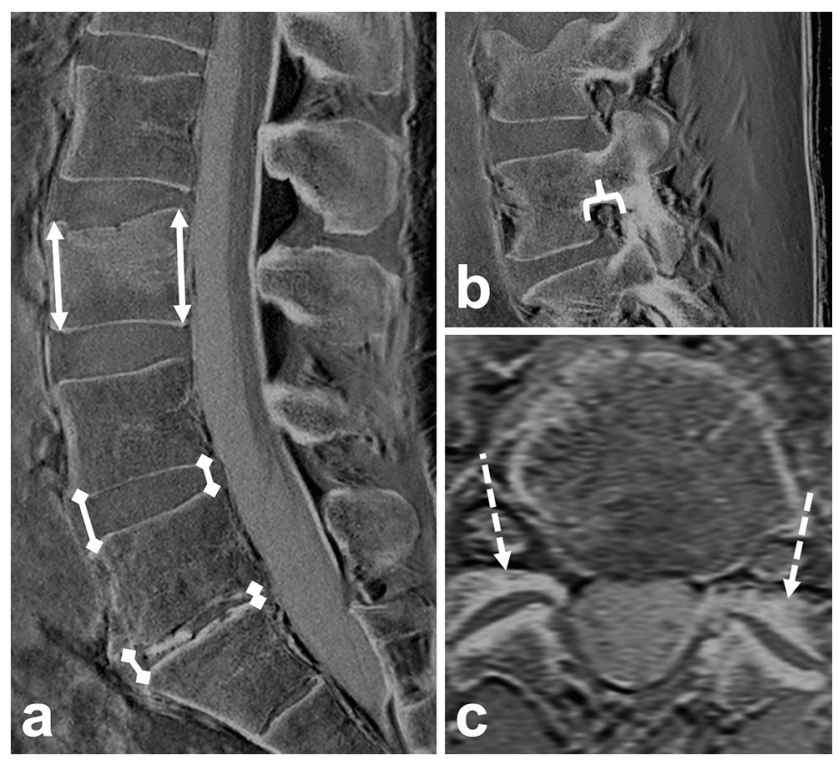

Fig. 3 Exemplary measurements in a 63-year-old male patient with an acute wedge-compression fracture of L3: Anterior and posterior height of the vertebral body (a; arrows); anterior and posterior height of intervertebral discs (a; arrows with rhomboid tips); neuroforaminal AP diameter (b; bracket); extent of facet joint degeneration (here: joint space narrowing + sclerosis; c; dashed arrows)

edema-like signal alterations on STIR as well as clinical symptoms. Fractures were most often found in L1 and L2 (each, $n=10)$, followed by Th12 $(n=9)$, L3 $(n=6)$, and the remaining thoracolumbar vertebral bodies. According to $\mathrm{CT}$ as the standard of reference, the majority of fractures were classified as wedge-compression fractures $(\mathrm{AO} \mathrm{A} 1 ; 68 \%)$ and incomplete and complete burst fractures (AO A3 + 4; 30\%; Table 2). Degenerative changes were assessed in two nonfractured vertebral segments in each patient $(n=60)$ and ranged from "no degenerative changes present" to "severe degenerative changes present" (Table 2).

The median for rating diagnostic quality of T1SGREderived images was 5 (excellent), with $59 \%$ of cases rated as excellent, $24 \%$ as good, $10 \%$ as moderate, $7 \%$ as poor, and none as inadequate (Figs. 1 and 2). The diagnostic quality of UTE images was rated significantly lower (median, 3 (moderate); with $45 \%$ of cases rated as good, $31 \%$ as moderate, and $24 \%$ as poor; $p<0.001$ ). The median for rating diagnostic quality of conventional CT images was 5 (excellent), with $82 \%$ of cases rated as excellent, and $17 \%$ as good.

\section{Diagnostic performance of MRI and agreement of MR and $\mathrm{CT}$ images}

On the T1SGRE sequence, 42 (reader 1) and 41 (reader 2) of 44 fractures were detected, using CT as standard of reference, while 3 (reader 1 ) and 3 (reader 2 ) fractures were considered to be false-positive vertebral fractures (sensitivity, 0.95/0.93; specificity, 0.98/0.98; accuracy 0.97/0.97 for radiologist 1 and 2, respectively). On the UTE sequence, 40 (reader 1) and 38 vertebral fractures (reader 2 ) were correctly identified, and 5 (reader 1 ) and 6 vertebral fractures (reader 2) were falsepositive vertebral fractures (sensitivity, $0.91 / 0.86$; specificity, $0.96 / 0.96$; accuracy $0.95 / 0.94$ for radiologist 1 and 2 , respectively).

Between T1SGRE-derived CT-like images and CT, agreement for quantitative parameters such as anterior and posterior heights of the vertebral body was excellent (ICCs, 0.99 [0.991.00], respectively (both radiologists); Table 3, Fig. 4). The agreement for ordinal-scale parameters was excellent as well, ranging between $\kappa 0.81(0.71-0.92)$ for fracture age (radiologist 2) and $\kappa 0.92(0.83-1.00)$ for Genant classification (both radiologists; Table 3). For quantitative parameters describing degenerative changes, agreement was excellent, ranging between ICC $0.81(0.62-0.90)$ for neuroforaminal AP diameter (radiologist 2) and $1.00(1.00-1.00)$ for spondylolisthesis (both radiologists; Table 3, Fig. 4). The agreement for ordinal-scale parameters ranged between substantial (sclerosis; $\kappa, 0.64$ [0.46-0.81] (radiologist 2)) and excellent (facet joint degeneration; ICC 0.96 [0.90-1.00] (radiologist 1); Table 3).

Between UTE images and CT, agreement for quantitative parameters was generally lower, with ICCs ranging from 0.79 (0.61-0.88) (neuroforaminal AP diameter; radiologist 2) to 0.99 (0.99-1.00) (spondylolisthesis; radiologist 1; Table 3, Fig. 4). Analogously, agreement between UTE images and CT for ordinal-scale parameters was generally lower, with $\kappa$ ranging between $0.43(0.26-0.60)$ (sclerosis; radiologist 2) and $0.89(0.79-0.99)$ (Genant classification; radiologist 1).

Inter-reader agreement ranged between substantial (sclerosis as evaluated on UTE images; $\kappa, 0.52$ [0.60-0.90]) and excellent (e.g., posterior height of the vertebral body evaluated on T1SGRE images and CT; ICCs, 0.99 [0.99-1.00], respectively; Table 4).

\section{Discussion}

In this analysis, we found a substantial to perfect diagnostic performance of T1SGRE-derived CT-like images and UTE with conventional $\mathrm{CT}$ for the identification of vertebral fractures. For the morphological assessment of fractures and degenerative bone changes, a substantial to perfect agreement was found as well as a robust image quality. Diagnostic performance of UTE, agreement between UTE and CT, and diagnostic image quality of UTE were generally lower. Inter-observer agreement was substantial-to-perfect for all modalities. Currently, patients with pathologies of the spine are regularly examined with CT and MR imaging to evaluate osseous and soft-tissue components of degenerative changes and fractures. For patients as well as from an economic perspective, it would be desirable to acquire all 
Table 3 Agreement of imaging findings between MR-derived and conventional CT

\begin{tabular}{|c|c|c|c|c|}
\hline \multirow[t]{2}{*}{ Parameter } & \multicolumn{2}{|c|}{ T1SGRE and conventional CT } & \multicolumn{2}{|c|}{ UTE and conventional CT } \\
\hline & Radiologist 1 & Radiologist 2 & Radiologist 1 & Radiologist 2 \\
\hline \multicolumn{5}{|l|}{ Fractures } \\
\hline Genant classification $(\kappa)$ & $0.92[0.83-1.00]$ & $0.92[0.83-1.00]$ & $0.89[0.79-0.99]$ & $0.69[0.52-0.85]$ \\
\hline Anterior height vertebral body (ICC) & $0.99[0.99-1.00]$ & $0.99[0.99-1.00]$ & $0.90[0.81-0.95]$ & $0.88[0.78-0.94]$ \\
\hline Posterior height vertebral body (ICC) & $0.99[0.99-1.00]$ & $0.99[0.99-1.00]$ & $0.89[0.80-0.94]$ & $0.90[0.80-0.94]$ \\
\hline $\mathrm{AO} /$ Magerl fracture classification $(\kappa)$ & $0.90[0.76-1.00]$ & $0.89[0.75-1.00]$ & $0.78[0.58-0.98]$ & $0.70[0.49-0.92]$ \\
\hline Fracture age $(\kappa)$ & $0.87[0.78-0.96]$ & $0.81[0.71-0.92]$ & $0.66[0.53-0.80]$ & $0.64[0.49-0.79]$ \\
\hline \multicolumn{5}{|l|}{ Degenerative changes } \\
\hline Anterior disc height (ICC) & $0.99[0.98-0.92]$ & $0.98[0.97-0.99]$ & $0.95[0.92-0.97]$ & $0.97[0.94-0.98]$ \\
\hline Posterior disc height (ICC) & $0.97[0.95-0.98]$ & $0.99[0.97-0.99]$ & $0.96[0.93-0.97]$ & $0.96[0.93-0.98]$ \\
\hline Neuroforamen AP diameter (ICC) & $0.95[0.89-0-97]$ & $0.81[0.62-0.90]$ & $0.91[0.85-0.95]$ & $0.79[0.61-0.88]$ \\
\hline Spondylolisthesis (ICC) & $0.99[0.99-1.00]$ & $0.99[0.99-1.00]$ & $0.99[0.99-1.00]$ & $0.98[0.97-0.99]$ \\
\hline Osteophytes (K) & $0.91[0.82-1.00]$ & $0.87[0.78-0.97]$ & $0.78[066-0.90]$ & $0.73[0.59-0.87]$ \\
\hline Sclerosis $(\kappa)$ & $0.68[0.48-0.88]$ & $0.64[0.46-0.81]$ & $0.52[0.32-0.72]$ & $0.43[0.26-0.60]$ \\
\hline Facet joint degeneration $(\kappa)$ & $0.96[0.90-1.00]$ & $0.88[0.78-0.97]$ & $0.66[0.48-0.84]$ & $0.67[0.53-0.81]$ \\
\hline
\end{tabular}

Data are given with 95\% confidence intervals. $\kappa$, weighted Cohen's kappa; ICC, intraclass correlation coefficient

Fig. 4 Bland-Altman plots for agreement between quantitative measurements on T1SGRE/ UTE and $\mathrm{CT}$ images, respectively. Measurements on T1SGRE vs. $\mathrm{CT}$ images are shown as black dots, and upper and lower limits of agreement are marked with fine dashed lines, respectively.

Measurements on UTE vs. CT images are shown as white rhombi, and upper and lower limits of agreement are marked with alternately dashed and dotted lines, respectively
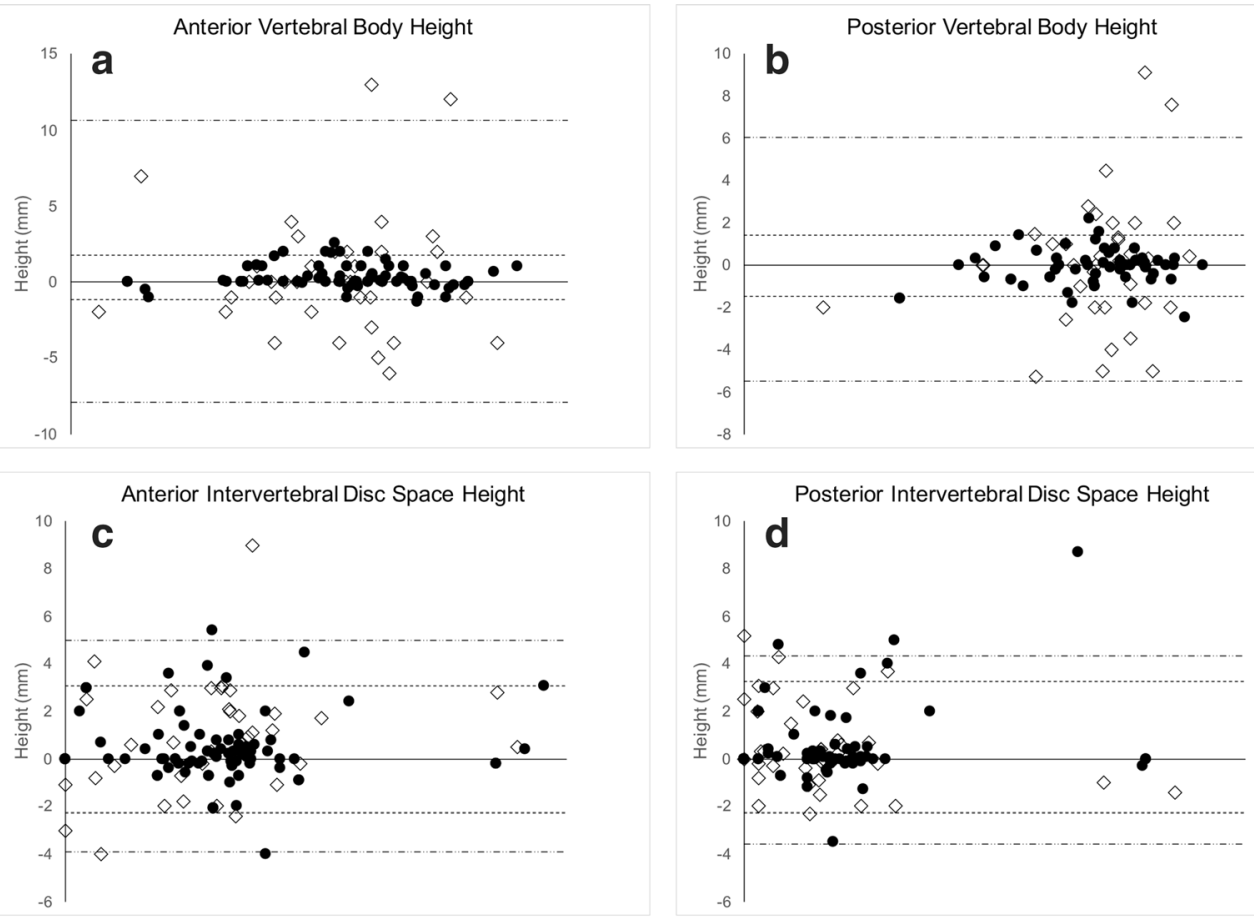

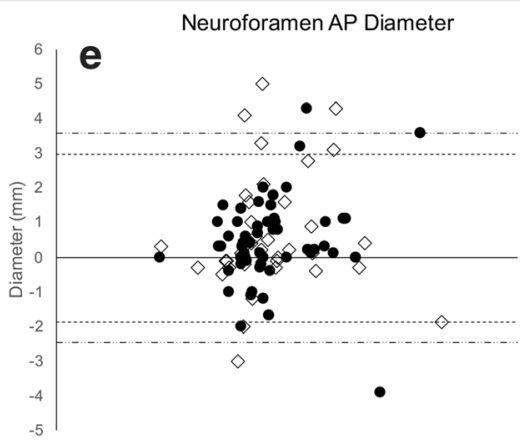

- T1SGRE vs. CT $\diamond$ UTE vs. CT 
Table 4 Inter-reader agreement of imaging findings between radiologist 1 and 2

\begin{tabular}{llll}
\hline Parameter & T1SGRE & Conventional CT & UTE \\
\hline Fractures & & & \\
Genant classification $(\kappa)$ & $0.95[0.87-1.00]$ & $0.95[0.87-1.00]$ & $0.77[0.63-0.92]$ \\
Anterior height vertebral body (ICC) & $0.98[0.96-0.99]$ & $0.98[0.96-0.99]$ & $0.97[0.95-0.99]$ \\
Posterior height vertebral body (ICC) & $0.99[0.99-1.00]$ & $0.99[0.99-1.00]$ & $0.99[0.98-0.99]$ \\
AO/Magerl fracture classification $(\kappa)$ & $0.81[0.63-0.99]$ & $0.95[0.84-1.00]$ & $0.77[0.56-0.98]$ \\
Fracture age $(\kappa)$ & $0.85[0.76-0.95]$ & $0.95[0.85-1.00]$ & $0.81[0.69-0.93]$ \\
Degenerative changes & & & \\
Anterior disc height (ICC) & $0.99[0.98-0.99]$ & $0.99[0.97-0.99]$ & $0.98[0.96-0.99]$ \\
Posterior disc height (ICC) & $0.98[0.97-0.99]$ & $0.98[0.96-0.99]$ & $0.97[0.95-0.98]$ \\
Neuroforamen AP diameter $($ ICC) & $0.93[0.88-0.96]$ & $0.87[0.76-0.93]$ & $0.98[0.97-0.99]$ \\
Spondylolisthesis $(\mathrm{ICC})$ & $1.00[1.00-1.00]$ & $1.00[1.00-1.00]$ & $0.99[098-0.99]$ \\
Osteophytes $(\kappa)$ & $0.82[0.71-0.93]$ & $0.83[0.71-0.95]$ & $0.81[0.69-0.93]$ \\
Sclerosis $(\kappa)$ & $0.75[0.60-0.90]$ & $0.79[0.67-0.91]$ & $0.52[0.29-0.75]$ \\
Facet joint degeneration $(\kappa)$ & $0.83[0.73-0.94]$ & $0.91[0.84-1.00]$ & $0.69[0.53-0.86]$ \\
\hline
\end{tabular}

Data are given with $95 \%$ confidence intervals. $\kappa$, weighted Cohen's kappa; ICC, intraclass correlation coefficient information in one examination. The CT-like images based on the T1SGRE sequence could enable MRI to reliably assess bone changes.

In particular, distance measurements were almost identical to measurements on conventional CT. Also, critical categorical variables such as the Genant and the $\mathrm{AO} /$ Magerl classifications showed an excellent agreement. By contrast, the extent of diffuse sclerosis still agreed substantially between T1SGRE-derived and conventional CT images, but this finding was affected by the tissue contrast on the T1SGRE sequence: Edema-like signal changes as occurring e.g. in Modic I changes induce a T1w signal decrease, and on intensity-inverted images, this might be indiscernible from sclerotic bone changes. Images should therefore always be read in combination with a fluid-sensitive pulse sequence.

Similarly, T1SGRE is not able to differentiate between bone and ligaments which both appear bright on grayscaleinverted reformatted images, as seen in Fig. 2. This must not be misinterpreted as ligament calcifications, and in the same context, the sensitivity of the proposed method for the assessment of ligament ossifications occurring e.g. in diffuse idiopathic skeletal hyperostosis must be expected to be low. Finally, gas accumulations in the intervertebral disc ("vacuum phenomenon") are devoid of signal and thus, bright on inverted reformatted images, not to be misinterpreted as calcifications.

The agreement between UTE and CT was lower for all assessed parameters, as well as the diagnostic image quality. Previously, UTE imaging was used to assess cortical bone in specimens [19], and trabecular bone in volunteers [18]. While showing convincing SNR in volunteers, the acquisition duration was $>9$ min. In another study assessing simulated spondylolysis in cadaveric spine specimens, diagnostic confidence of UTE imaging was comparable to CT [32]. By contrast, the study presented here is based on clinical subjects. SNR and signal homogeneity in the thoracolumbar spine were not optimal, and UTE was prone to pulsation and movement artefacts. Moreover, UTE as a non-Cartesian imaging method is sensitive to off-resonance blurring induced by $\mathrm{B}_{0}$ inhomogeneities and fat chemical shift. Most importantly, the presently employed UTE imaging protocol needed a large field-ofview and substantial oversampling to avoid folding and the slice thickness was twice the thickness of T1SGRE to maintain acceptable acquisition durations. To maintain a primarily PD-weighted contrast, a small flip angle had to be used which reduced SNR. Also, to achieve an acceptable acquisition duration and advanced methods of improving short T2 contrast such as inversion recovery or subtraction methods were not used here. The use of longT2 suppression techniques has to be evaluated in the future. Of note, UTE imaging has potential advantages regarding tissue contrast: It may be highly useful e.g. for assessing the cartilage endplate [33] and to differentiate ligaments and calcified structures. ZTE pulse sequences, on the other hand, generate real PD-weighted tissue contrast and have been successfully applied to the shoulder, hip, skull, and cervical spine [20, 34-36]. How they perform in the thoracolumbar spine in comparison to T1SGRE should be evaluated in future studies.

Particularly in the context of emergency care, MRI compared to CT is usually less accessible, associated with higher costs, longer examination duration and requires a good patient compliance. Therefore, the application of the proposed method may be limited to centers with necessary resources and/ or to patients which will undergo an MRI examination in any case. 
However, it has to be noted that conventional CT, which served as the standard of reference for the assessment of morphologic bone changes here, may miss "occult vertebral fractures", i.e., traumatic injuries without significant morphologic changes but with the presence of a "bone bruise", i.e. edema-equivalent signal changes in the bone marrow that can be detected on fluid-sensitive MRI sequences [37, 38]. In this study, no patient with this trauma pattern was included, but it may be assumed that the combination of clinically established MRI sequences including STIR and the proposed sequences for dedicated bone assessment may be the most sensitive sensible option for the detection of vertebral injuries.

This study has limitations. First, the acquisition of the proposed gradient duty-cycle intensive pulse sequences requires a 3-T MR scanner with state-of-the-art gradient coils. On older scanners, examination duration might be prolonged due to increased minimum TR. Furthermore, particularly T1SGRE and less so UTE are sensitive to metal artifacts and thus, in patients with metallic implants, CT or CT myelography may still be the preferred diagnostic method.

Moreover, no patients with more severe fracture patterns, i.e., distraction and translation injuries, were included in this study, since these patients regularly undergo surgery immediately after CT. Since all morphometric parameters assessed here showed a substantial to perfect agreement, it could be assumed that this would apply to more severe injury patterns just as well; however, this needs to be evaluated in future studies. Analogously, in this first assessment, we did not include patients with pathologic fractures or bone metastases. Whether or not the used sequences are equivalent to $\mathrm{CT}$ or may even add diagnostic value in these cases (due to perfect alignment with other MR sequences in the protocol) has yet to be evaluated.

In summary, CT-like images obtained from a T1SGRE sequence showed a substantial to perfect agreement with conventional $\mathrm{CT}$ for the assessment of vertebral fractures and degenerative bony changes. Agreement between UTE imaging and CT was substantial but generally lower due to primarily less robust UTE image quality. Therefore, in specific cases, adding the T1SGRE sequence to spine MR examination protocols could render additional CT examinations obsolete in the future, spare additional examinations, and reduce radiation doses and costs.

Funding Open Access funding enabled and organized by Projekt DEAL. The authors state that this work has not received any funding.

\section{Compliance with ethical standards}

Guarantor The scientific guarantor of this publication is Benedikt J. Schwaiger, MD.
Conflict of interest The authors of this manuscript declare no relationships with any companies whose products or services may be related to the subject matter of the article.

Statistics and biometry One of the authors has significant statistical expertise (Benedikt J. Schwaiger, 11 years of experience in biostatistics).

Informed consent Written informed consent was obtained from all subjects (patients) in this study.

Ethical approval Institutional Review Board approval was obtained.

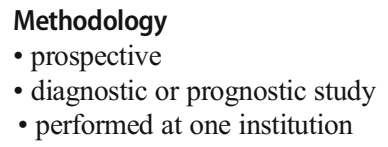

Open Access This article is licensed under a Creative Commons Attribution 4.0 International License, which permits use, sharing, adaptation, distribution and reproduction in any medium or format, as long as you give appropriate credit to the original author(s) and the source, provide a link to the Creative Commons licence, and indicate if changes were made. The images or other third party material in this article are included in the article's Creative Commons licence, unless indicated otherwise in a credit line to the material. If material is not included in the article's Creative Commons licence and your intended use is not permitted by statutory regulation or exceeds the permitted use, you will need to obtain permission directly from the copyright holder. To view a copy of this licence, visit http://creativecommons.org/licenses/by/4.0/.

\section{References}

1. Burton AK, Balague F, Cardon G et al (2006) Chapter 2. European guidelines for prevention in low back pain: November 2004. Eur Spine J 15(Suppl 2):S136-S168

2. Airaksinen O, Brox JI, Cedraschi C et al (2006) Chapter 4. European guidelines for the management of chronic nonspecific low back pain. Eur Spine J 15(Suppl 2):S192-S300

3. Palmer KT, Walsh K, Bendall H, Cooper C, Coggon D (2000) Back pain in Britain: comparison of two prevalence surveys at an interval of 10 years. BMJ 320:1577-1578

4. Buchbinder R, Blyth FM, March LM, Brooks P, Woolf AD, Hoy DG (2013) Placing the global burden of low back pain in context. Best Pract Res Clin Rheumatol 27:575-589

5. Haussler B, Gothe H, Gol D, Glaeske G, Pientka L, Felsenberg D (2007) Epidemiology, treatment and costs of osteoporosis in Germany-the BoneEVA Study. Osteoporos Int 18:77-84

6. Hernlund E, Svedbom A, Ivergard M et al (2013) Osteoporosis in the European Union: medical management, epidemiology and economic burden. A report prepared in collaboration with the International Osteoporosis Foundation (IOF) and the European Federation of Pharmaceutical Industry Associations (EFPIA). Arch Osteoporos 8:136

7. Bliuc D, Nguyen ND, Milch VE, Nguyen TV, Eisman JA, Center JR (2009) Mortality risk associated with low-trauma osteoporotic fracture and subsequent fracture in men and women. JAMA 301: $513-521$

8. Ioannidis G, Papaioannou A, Hopman WM et al (2009) Relation between fractures and mortality: results from the Canadian Multicentre Osteoporosis Study. CMAJ 181:265-271

9. Shanechi AM, Kiczek M, Khan M, Jindal G (2019) Spine anatomy imaging: an update. Neuroimaging Clin N Am 29:461-480 
10. Mandalia V, Henson JH (2008) Traumatic bone bruising-a review article. Eur J Radiol 67:54-61

11. Piazzolla A, Solarino G, Lamartina C et al (2015) Vertebral bone marrow edema (VBME) in conservatively treated acute vertebral compression fractures (VCFs): evolution and clinical correlations. Spine 40:E842-E848

12. Schegerer A, Loose R, Heuser LJ, Brix G (2019) Diagnostic Reference Levels for Diagnostic and Interventional X-Ray Procedures in Germany: Update and Handling. RoFo 191:739-751

13. Sistrom CL, McKay NL (2005) Costs, charges, and revenues for hospital diagnostic imaging procedures: differences by modality and hospital characteristics. J Am Coll Radiol 2:511-519

14. Lecouvet FE, Pasoglou V, Van Nieuwenhove S et al (2020) Shortening the acquisition time of whole-body MRI: 3D T1 gradient echo Dixon vs fast spin echo for metastatic screening in prostate cancer. Eur Radiol 30:3083-3093

15. Lee RK, Griffith JF, Tong MM, Sharma N, Yung P (2013) Glenoid bone loss: assessment with MR imaging. Radiology 267:496-502

16. Nordeck SM, Koerper CE, Adler A et al (2017) Simulated radiographic bone and joint modeling from 3D ankle MRI: feasibility and comparison with radiographs and 2D MRI. Skeletal Radiol 46: 651-664

17. Gersing AS, Pfeiffer D, Kopp FK et al (2019) Evaluation of MRderived CT-like images and simulated radiographs compared to conventional radiography in patients with benign and malignant bone tumors. Eur Radiol 29:13-21

18. Ma YJ, Chen Y, Li L et al (2020) Trabecular bone imaging using a 3D adiabatic inversion recovery prepared ultrashort TE Cones sequence at 3 T. Magn Reson Med 83:1640-1651

19. Lu X, Jerban S, Wan L et al (2019) Three-dimensional ultrashort echo time imaging with tricomponent analysis for human cortical bone. Magn Reson Med 82:348-355

20. Wiesinger F, Sacolick LI, Menini A et al (2016) Zero TE MR bone imaging in the head. Magn Reson Med 75:107-114

21. Chang EY, Du J, Chung CB (2015) UTE imaging in the musculoskeletal system. J Magn Reson Imaging 41:870-883

22. Qian Y, Boada FE (2008) Acquisition-weighted stack of spirals for fast high-resolution three-dimensional ultra-short echo time MR imaging. Magn Reson Med 60:135-145

23. Genant HK, Wu CY, van Kuijk C, Nevitt MC (1993) Vertebral fracture assessment using a semiquantitative technique. J Bone Miner Res 8:1137-1148

24. Magerl F, Aebi M, Gertzbein SD, Harms J, Nazarian S (1994) A comprehensive classification of thoracic and lumbar injuries. Eur Spine J 3:184-201

25. Vaccaro AR, Oner C, Kepler CK et al (2013) AOSpine thoracolumbar spine injury classification system: fracture description, neurological status, and key modifiers. Spine 38:2028-2037
26. Hedderich DM, Maegerlein C, Baum T et al (2019) Differentiation of acute/subacute versus old vertebral fractures in multislice detector computed tomography: is magnetic resonance imaging always needed? World Neurosurg 122:e676-e683

27. Frobin W, Brinckmann P, Kramer M, Hartwig E (2001) Height of lumbar discs measured from radiographs compared with degeneration and height classified from MR images. Eur Radiol 11:263-269

28. Mamisch N, Brumann M, Hodler J et al (2012) Radiologic criteria for the diagnosis of spinal stenosis: results of a Delphi survey. Radiology 264:174-179

29. Wilke HJ, Rohlmann F, Neidlinger-Wilke C, Werner K, Claes L, Kettler A (2006) Validity and interobserver agreement of a new radiographic grading system for intervertebral disc degeneration: Part I. Lumbar spine. Eur Spine J 15:720-730

30. Weishaupt D, Zanetti M, Boos N, Hodler J (1999) MR imaging and CT in osteoarthritis of the lumbar facet joints. Skeletal Radiol 28: 215-219

31. Cohen J (1968) Weighted kappa: nominal scale agreement with provision for scaled disagreement or partial credit. Psychol Bull 70:213-220

32. Finkenstaedt T, Siriwanarangsun $P$, Achar $S$ et al (2019) Ultrashort time-to-echo magnetic resonance imaging at $3 \mathrm{~T}$ for the detection of spondylolysis in cadaveric spines: comparison with $\mathrm{CT}$. Invest Radiol 54:32-38

33. Berg-Johansen B, Han M, Fields AJ et al (2018) Cartilage endplate thickness variation measured by ultrashort echo-time MRI is associated with adjacent disc degeneration. Spine 43:E592-E600

34. Breighner RE, Bogner EA, Lee SC, Koff MF, Potter HG (2019) Evaluation of osseous morphology of the hip using zero echo time magnetic resonance imaging. Am J Sports Med 47:3460-3468

35. Breighner RE, Endo Y, Konin GP, Gulotta LV, Koff MF, Potter HG (2018) Technical developments: zero echo time imaging of the shoulder: enhanced osseous detail by using MR imaging. Radiology 286:960-966

36. Argentieri EC, Koff MF, Breighner RE, Endo Y, Shah PH, Sneag DB (2018) Diagnostic accuracy of zero-echo time MRI for the evaluation of cervical neural foraminal stenosis. Spine 43:928-933

37. Qaiyum M, Tyrrell PN, McCall IW, Cassar-Pullicino VN (2001) MRI detection of unsuspected vertebral injury in acute spinal trauma: incidence and significance. Skeletal Radiol 30:299-304

38. Yao L, Lee JK (1988) Occult intraosseous fracture: detection with MR imaging. Radiology 167:749-751

Publisher's note Springer Nature remains neutral with regard to jurisdictional claims in published maps and institutional affiliations. 